\title{
MASTER SLAVE AUTONOMOUS SURVEILLANCE BOT FOR MILITARY APPLICATIONS
}

\author{
Rajath Kumar M.P ${ }^{1}$, Nishanth N Bhonsle ${ }^{2}$, Nitin R Hugar ${ }^{3}$ \\ ${ }^{I}$ Department of Electronics and Communication Engineering, R. N. Shetty Institute of Technology (RNSIT), \\ Bangalore, India \\ ${ }^{2}$ Department of Electronics and Communication Engineering, R. N. Shetty Institute of Technology (RNSIT), \\ Bangalore, India \\ ${ }^{3}$ Department of Electronics and Communication Engineering, R. N. Shetty Institute of Technology (RNSIT), \\ Bangalore, India
}

\begin{abstract}
Defense and surveillance are some of the most important aspects in today's society. In a place where knowledge translates to power, having a little more information can make a lot of difference in the outcome of any conflict. Therefore, the military is always looking for new technologies to improve their preparedness for any event. Knowing their surroundings and the battlefield well beforehand can turn the tables on the battlefront and can possibly save lives. In this paper, the protocol for detection and wireless transmission of this data has been illustrated and the various problem statements regarding locomotion, ranging, defense, temperature, moisture and various other parameters have been resolved and implemented. The intercommunication between master and slave bots is demonstrated and used to transmit and receive data and instructions to one another. The bots perform tasks that would normally be too extreme for humans to do. All the information is also stored as a database while some of the data can also be published for the information of the public.
\end{abstract}

Keywords - Defense, surveillance, sensors communication, master-slave robots

\section{INTRODUCTION}

Robots are being adopted widely in almost all domains. Recent outburst in the use of robots in the military has drawn a lot of attention towards the research and testing of defense equipment and it is now a rapidly growing technological field. Tasks like mine detection or neutralization of live explosives put human lives at stake. A lot of robots are currently being utilized in military reconnaissance, geomapping, planetary exploration, human detection and in many mission based services[7]. Our environment is hostile and unpredictable and thus, the need for unmanned surveillance is acute in geographically difficult regions[2]. Determination of the feasibility of living, the prevailing ambient environmental conditions, terrain mapping, wireless communicability are some of the prime factors that need to be focused upon[9]. The inter-robot communication link enables a quick exchange of information without human intervention, this facilitates a more reliable result[3].

\section{SENSORS}

\subsection{LM32}

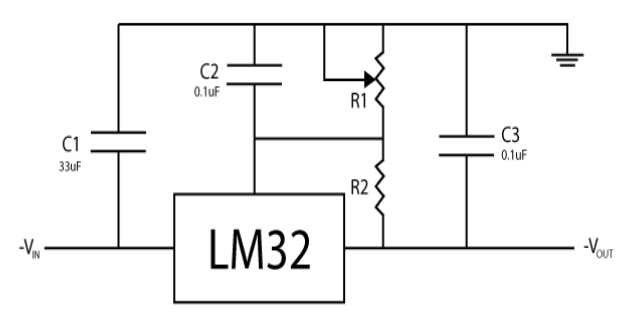

Fig1. LM32 circuit diagram

LM32 is a dual thermal diode temperature sensor with sensor path TM bus and it is a digital temperature sensor. It measures 3 temperature zones and has a single-wired interface compatible with National Semiconductor's Path bus.

LM32 is pulse width encoded and hence it can be connected to all general purpose microcontrollers. It measures the temperature of its own die and two external devices like a diode connected transistor and a processor thermal diode and it can resolve temperatures to $255^{\circ} \mathrm{C}$ and down to $256^{\circ} \mathrm{C}$. The temperature is being measured by monitoring the change in resistance as per the change in temperature. 
LM32 can be used to give a high output when the temperature exceeds a particular temperature value as well. LM32 eliminates the need of A/D converter and its output is precise and is a balanced digital reading.

\subsection{HC-SR04}

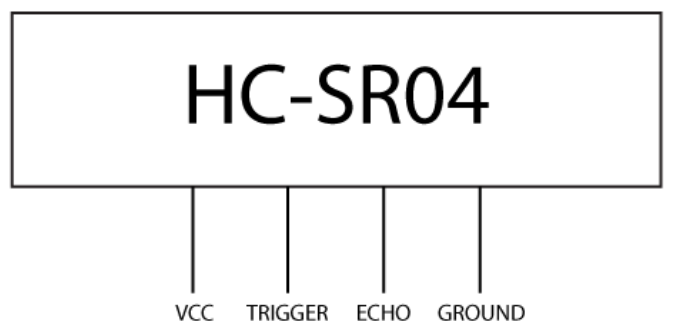

Fig2. HC-SR04 Pin Diagram

HC-SR04 is an ultrasonic sensor that emits high frequency short length sound pulses at regular intervals. When these pulses strike an object, they get reflected back as echo signals and the sensor computes the target based on the time span between the receiving echo and the transmitting pulse[8]. These sensors are excellent in suppressing background interference as time of flight is considered rather than the intensity of sound. Ultrasonic sensor detects all kinds of materials and even transparent materials and thin foils also won't cause any problem in detection. Ultrasonic sensors can see through dust-laden air and ink mists and even thin deposits on the sensor membrane do not impair its function. The maximum range is $4 \mathrm{~m}$ and minimum is $2 \mathrm{~cm}$.

\subsection{LM393}

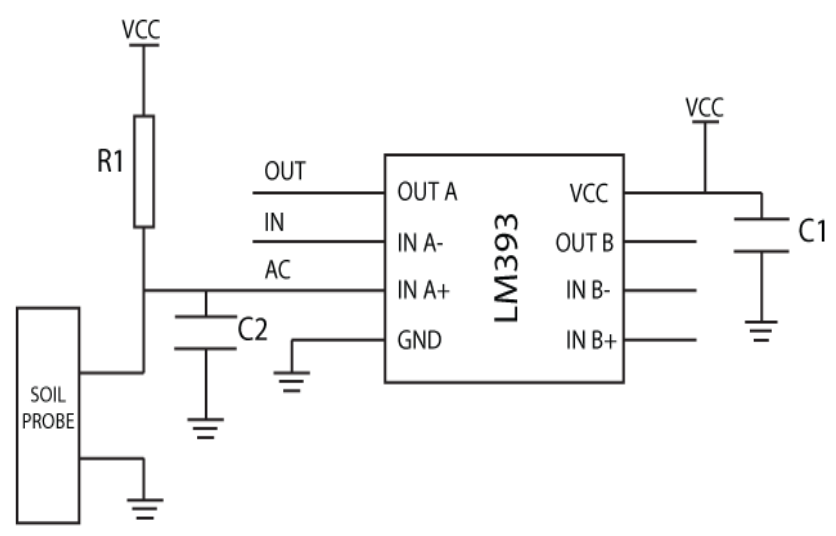

Fig3. LM393 Circuit Diagram

LM393 is a simple moisture and humidity sensor used to detect soil moisture or the water content in the soil. It is made up of multiple moisture sensors and it measures the moisture by considering parameters such as electrical resistance or interaction with neurons, as a proxy for moisture content. The sensor goes High when the soil moisture is deficit and vice versa. The sensitivity is adjustable and its operating voltage is 3.3-5V.This module works on an analog output mode which gives scalable analog readings using which the moisture and humidity is calculated.

\subsection{L293D}

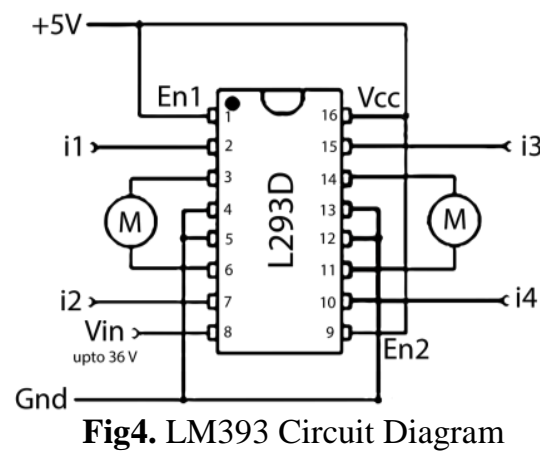

L293D is a dual h-bridge motor driver integrated circuit. Motor drivers act as current amplifier circuit as they take a low-current signal and provide higher-current signal. This higher current signal is used to drive motors. L293D contains two inbuilt $\mathrm{H}$-bridge driver circuits. The common mode of operation consists of two DC motors that can be driven simultaneously in forward and reverse direction. The motor operations can be controlled by input logic combinations. 00 or 11 will stop the corresponding motor. 01 and 10 will rotate it clockwise and anticlockwise, respectively. Enable pins on the development boards of the corresponding motors must be high for motors to start operating. Enable input being high, the associated driver is enabled. Thus, the outputs become active and work in phase with their inputs. When the enable input is low, driver is disabled, and their outputs are off.

\section{DEVELOPMENT BOARDS}

\subsection{Atmega328}

This microcontroller is based on Atmel 8-bit AVR RISC. It comes with 23 general purpose I/O lines, $1 \mathrm{~KB}$ EEPROM,2KB

SRAM, $32 \mathrm{~KB}$ ISP flash memory, 32 general purpose registers, 3 timer/counters, internal and external interrupts, SPI serial port, 6-channel 10-bit A/D converter, serial programmable USART, 5 programmable selectable power saving modes, 2-wire serial interface with byte orientation, programmable watchdog timer with internal oscillator and it has read-while-write capabilities. The device operating voltage is between 1.8-5.5 volts.

\subsection{Broadcom BCM2835}

This System on Chip (SoC) is stacked under the RAM chip and includes ARM1176JZF-S processor operating at 700 $\mathrm{MHz}$, Video Core IV GPU and $512 \mathrm{MB}$ SDRAM. The Level 1 cache is $16 \mathrm{~KB}$ and Level 2 cache is $128 \mathrm{~KB}$. The GPU primarily uses the Level 2 cache. It comes with 4 USB 2.0 ports via integrated USB hub and LAN9512, HDMI video output, Micro Secure Digital Card slot for on board storage, 10/100 wired Ethernet RJ45 for on board network, 40 General Purpose Input-Output (GPIO) pins, Serial Peripheral Interface Bus (SPI), Universal asynchronous receiver/transmitter (UART). Power rating is approximately $650 \mathrm{~mA}$. 


\section{WORKING}

\subsection{Master Bot}

Powered by Broadcom BCM235, the master bot plays a vital role. The master bot controls the slave bot with an instruction set. It is interfaced with a camera module and wifi adaptor. For communication with slave bots, an RF transmitter and Receiver of $434 \mathrm{MHz}$ is interfaced[3]. It has on board storage SD card for storing the received data. Furthermore, it is pre-programmed and data is pre-loaded for autonomous functioning of the bot.

\subsection{Social Network}

Social Networking site, Twitter provides an API to make Twitter-bots, which would enable the master bot to tweet updates or the data it receives from the slave bot. Tweepy is the library that is used [1]. This library is a python library and has built in functions to tweet to the account. At first, the application has to be registered with twitter which provides Consumer API key and Access token which has to be fed into a python script which has Tweepy library imported. A python script is written which enables the bot to publish real time data that it is being fetched when on a operation/mission. The data might be a picture taken from the camera module attached to the bot or readings from the various sensors that is present in the sensor bot.

\subsection{Slave Bot}

The slave bot is a rover interfaced with many necessary sensors for completing all required tasks and is controlled and monitored by Atmega328. It receives instructions from the master bot as to what parameter of the environment has to be scanned and reported to the master. Some of the main functions of this bot are to find out the scalable moisture, exact temperature and humidity parameters of the surrounding environment and also terrain mapping and ranging. The communication link between the slave and the master is established by either a Bluetooth module or a $434 \mathrm{MHz}$ transceiver. The master bot sends a particular code which represents the task to be done and reported. The Atmega328 is programmed in such a way that upon reception, the controller checks the ASCII code sent and performs the assigned task or function. The bot movements and sensor values are obtained by the following assigned codes :

\begin{tabular}{|c|c|l|}
\hline Value sent & $\begin{array}{l}\text { ASCII value } \\
\text { received }\end{array}$ & Task assigned \\
\hline (master) & (slave) & \\
\hline 1 & 49 & move forward \\
\hline 2 & 50 & move backward \\
\hline 3 & 51 & move right \\
\hline 4 & 52 & move left \\
\hline 5 & 53 & sense temperature \\
\hline 6 & 54 & $\begin{array}{l}\text { sense moisture and } \\
\text { humidity }\end{array}$ \\
\hline 7 & 55 & terrain mapping and ranging \\
\hline
\end{tabular}

\subsection{Metal Detector}

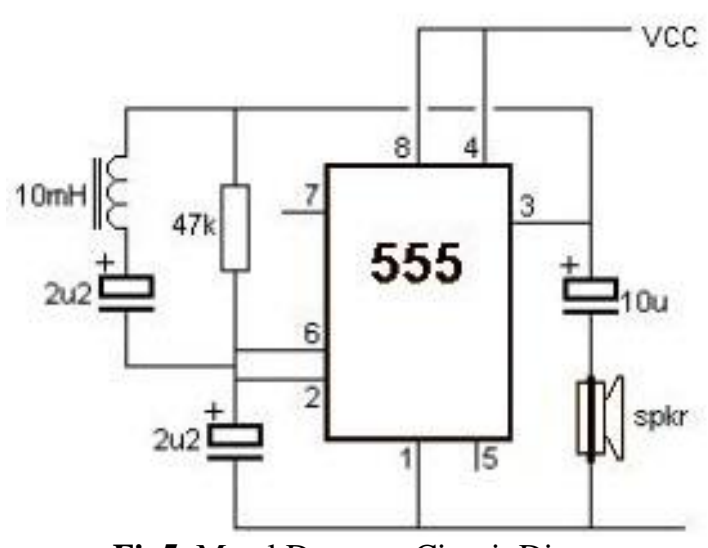

Fig5. Metal Detector Circuit Diagram

A simple metal detector circuit diagram is designed using IC 555, similar to the 555 timer circuits; these circuits detect the metals and magnets. When a magnet is near the choke (10mh) shown in the circuit, the o/p frequency changes. This circuit is powered with a power supply, which provides an $\mathrm{o} / \mathrm{p} \mathrm{DC}$ voltage between $6 \mathrm{~V}$ to $12 \mathrm{~V}$. If a metal is near the coil L1, it produces a change of o/p oscillation frequency, and then generates a buzzing sound.

\subsection{Communication}

\subsubsection{HC05}

HC-05 is a class-2 Bluetooth module which can be used on either the master or the slave as a drop-in replacement for wired serial communication. It can be used for a serial port replacement to establish connection between any Bluetooth enabled device and the robot. It operates at a frequency of $2.4 \mathrm{GHz}$ and Gaussian frequency shift keying (GFSK). It has a sensitivity of $-84 \mathrm{dBm}$ and works within a temperature range of -20 to +75 centigrade. if the transmission is asynchronous then it happens at a speed of $2.1 \mathrm{Mbps}$ and if it is synchronous then it happens at speed of $1 \mathrm{Mbps}$. It requires a +3.3VDC 50mA power supply for its operation.

\subsubsection{RF434 Transreceiver}

The RF434 operates at radio frequency and varies in the frequency range of $30 \mathrm{kHz}$ to $300 \mathrm{GHz}$. In this the digital data is amplitude modulated and transmitted over the carrier thus representing Amplitude Shift Keying (ASK). The RF transmission is better than IR as they can travel larger distances than the latter in long range applications. Also, RF signals can travel even when there is an obstruction between the transmitter and the receiver while IR is restricted to line of sight mode. RF is strong and more reliable and uses a specific frequency unlike IR which is likely to be affected by other IR emitting sources. The sensor used in this prototype operates at $434 \mathrm{MHz}$ and the transmitter receives serial data and transmits it wirelessly through RF through its antenna at the rate of $1 \mathrm{Kbps}-10 \mathrm{Kbps}$. The transmitted data is being received by the receiver operating at the same frequency as that of the transmitter. 


\section{BLOCK DIAGRAM}

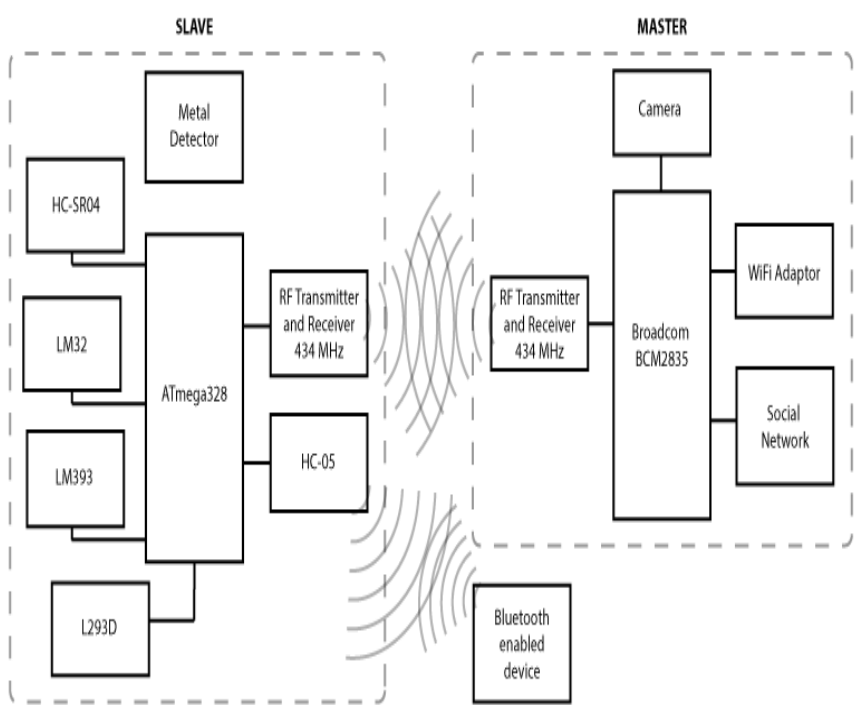

Fig6. Master Slave Proposed Block Diagram

\section{CALCULATIONS}

\subsection{LM32}

The input is read from the sensor and stored in the variable "Value".

$$
\text { value }=\text { input }
$$

This read value is converted into Celsius by using the formulae.

$$
\text { celsius }=\frac{\text { value }}{1024} \times 500
$$

Also Celsius value can be expressed in Fahrenheit as

$$
\text { fahrenheit }=\frac{\text { celsius } \times 9}{5}+32
$$

\subsection{HC-SR05}

A sound pulse signal and waits for 50 microseconds for the signal to return after echo. Then, it calculates the time taken for the completion of this process and stores this in variable "pulse time".

$$
\text { pulsetime }=\text { input }
$$

The speed of sound is $340 \mathrm{~m} / \mathrm{s}$ or $29 \mathrm{uS} / \mathrm{cm}$. Since the pulse signal travels out and back we divide by 58 to calculate the distance, D of the object.

$$
D=\frac{\text { pulsetime }}{58}
$$

This is repeated for 10 times and the total distance is sum total of all the distance that was calculated.

$$
\text { Total }=D+\text { Total }
$$

Average distance, AvgD is the sum total of all the distance divided by the number of times it was iterated in this case it is $10(\mathrm{n}=10)$.

$$
\operatorname{Avg} D=\frac{\text { Total }}{n}
$$

\section{RESULTS}

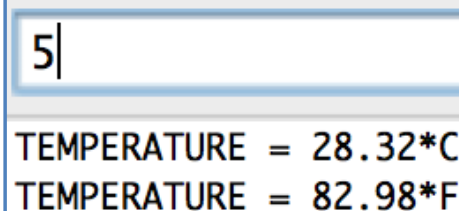

Fig7. LM32 Results

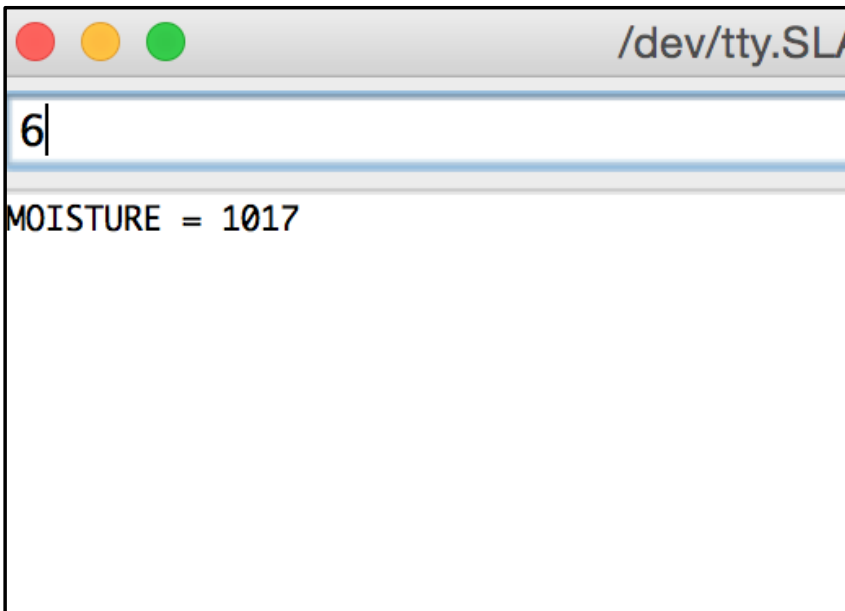

Fig8. LM393 Results

\begin{tabular}{|l|}
\hline 7 \\
\hline $243 \mathrm{~cm}$ \\
$223 \mathrm{~cm}$ \\
$244 \mathrm{~cm}$ \\
$243 \mathrm{~cm}$ \\
$260 \mathrm{~cm}$ \\
$264 \mathrm{~cm}$ \\
$223 \mathrm{~cm}$
\end{tabular}

Fig9. HC-SR04 Results 


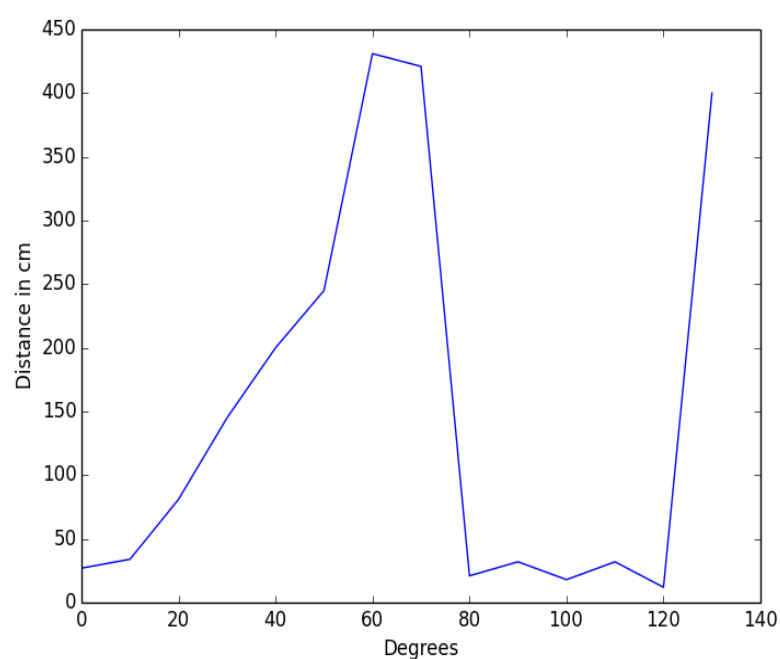

Fig10. Obstacle Distance v/s Degree Plot

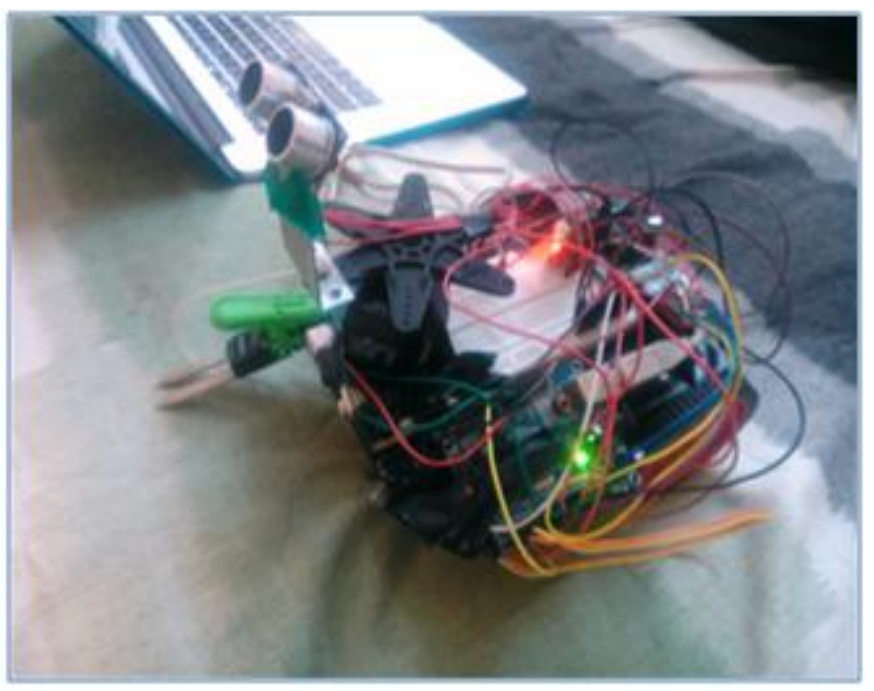

Fig11. Proposed Slave Bot

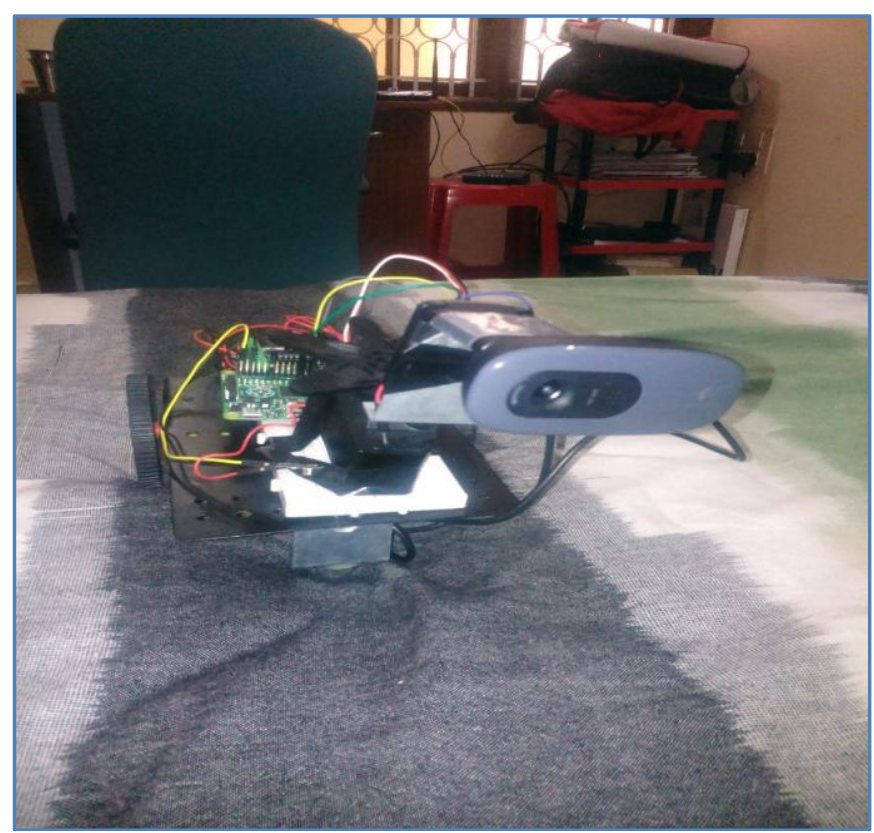

Fig12. Proposed Master Bot

\section{APPLICATIONS}

The bot is mainly used for remote area surveillance. It is a reliable source of information about all the parameters necessary to be determined. It enables the general public to get information in real time about the weather conditions of a particular place through social networking medium. It reduces the risks to humans as extreme tasks are carried out by this bot that would be dangerous for humans to do. It is robust and unlike humans, its efficiency is not dependent on environmental factors. It can be used for facial recognition which finds applications in law and order tasks. Navigation and geo mapping is also performed by this bot. The Project can find applications in more extreme situations like surveillance and mapping of the terrain of other planets to find water ambient temperature and sustainability of life on those planets. It can be public warning system during calamities or natural disasters, using social media to raise caution. Medical field can benefit from the use of the bots which can be modified to check and monitor the parameters such as body temperature water content, Blood pressure and pulse rate of the patients remotely thus eliminating the time lapse and allowing doctors to treat more patients at once.

\section{CONCLUSION}

Having artificial intelligence and robotic devices improves efficiency and ease of work in times of peril having robot do all the dangerous work while soldiers control it from a safe distance is the best case scenario. The amount of data provided by the robot gives the soldiers a wealth of information about the battle ground scenario and thus gives them a added advantage.

\section{REFERENCES}

[1]. Ren C Luo, Kuo L Su, Shen H Shen and Kuo H Tsai. "Networked Intelligent Robots Through The Internet Issues and oppurtunities " Proceedings of the IEEE, vol.91, no.3, pp.371,382, March 2003.

[2]. Dr. Michael C Wicks, Mr. John F Spina, Dr. Gerald T CapraroA. "Radar the Next generation - Sensor as Robots "Radar Conference, 2003 Proceedings of the International.

[3]. Wei - Hao Hsu, You - Ling Jian, Feng - Li Lian. "Multi - Robot Movement Design Using the Number of Communication Links "Systems, Man and Cybernetics,2006. SMC '06. IEEE International

[4]. Mr. Sandeep Bhat, Dr. M Meenakshi "Vision Based Robotic System for Military Applications - Design and Real Time Validation “ 2014 Fifth International conference on signal and image processing(ICSIP).

[5]. Rice K, Le Moigne J, Jain P. “Analyzing Range Maps Data for Future Space Robotics Applications "Space Mission challenges for Information Technology, 2006. SMC-IT 2006.

[6]. HoChung Chang, TaMing Shih. "Visual servo control of a three degree of freedom robotic arm system "Advanced robotics and its social impacts,2008.ARSO 2008.IEEE Workshop on vol.,no.,pp.1,6,2325 Aug 2008. 
[7]. Willis J B, Davis M J. "Design of the Reconnaissance, Surveillance, and Target Acquisition Squadron for the US Army's Interim Brigade Combat Team," Systems, Man, and Cybernetics, 2000 IEEE International Conference on , vol.1, no., pp.478,483 vol.1, 2000

[8]. J. Larson, M. Bruch, R Halterman, J Rogers, R Webster, "Advances in Autonomous Obstacle Avoidance for Unmanned Surface Vehicles", Space and Naval Warfare systems center San Diego CA, 2007.

[9]. M.H. Bruch, J. Lum, S. Yee, and N. Tran, "Advances in Autonomy for Small UGVs," SPIE Proc. 5804: Unmanned Ground Vehicle Technology VII, Orlando, FL, 2005, 29-31. [10]. M.H. Bruch, R.T. Laird, and H.R. Everett, "Challenges for deploying man-portable robots into hostile Environments," SPIE Proc. 4195: Mobile Robots XV, Boston, MA, 2000, 5-8. 